\title{
Generic adaptation mechanism for the support of context-aware service provision in 3G networks
}

\author{
Nikos Houssos, Spyros Pantazis, Athanassia Alonistioti \\ Communication Networks Laboratory, Department of Informatics \& Telecommunications, \\ University of Athens, 157 84, Athens, Greece, \\ email:\{nhoussos, pantazis, nancy\}@di.uoa.gr
}

\begin{abstract}
The forthcoming era of $3^{\text {rd }}$ generation (3G) mobile communications is heralded to change the wireless telecommunication experience dramatically. User expectations are raised to a significantly higher level, towards the demand for terminal-, network- and location-aware provision of ubiquitous, personalized multimedia services. Under this perspective, the need for adaptability of services and systems to largely diverse contexts is clearly identified. Generic, dynamically extensible adaptation mechanisms that are independent of the subject and criteria of adaptation can be a significant step towards this direction. In the present paper $^{1}$, we introduce a mechanism aiming to fulfill these requirements. The proposed adaptation system has been designed, implemented and successfully integrated and tested in a distributed software platform for provision of value added services to $3 \mathrm{G}$ mobile users.
\end{abstract}

Keywords: adaptability, context-awareness, 3G mobile services, service management platforms, reconfigurability, ubiquitous computing.

\section{INTRODUCTION}

The evolution of mobile networks and systems to $3^{\text {rd }}$ generation and beyond is expected to bring about substantial changes to telecommunication service provision. According to existing paradigms of $2-2.5 \mathrm{G}$, end-users are offered a small number of vertically integrated services that are tightly to specific standards and technologies. In the envisioned new era, however, where customer expectations as well as investments and revenue requirements are raised to a new level, a plethora of functionality-rich, profit-creating applications, typically contributed by many different business players, should be available to mobile users [1]. Moreover, services should be accessible in an unprecedented variety of contexts [2], which could not be predicted or catered for during service design and development. These extensively demanding requirements clearly indicate the need for adaptability of applications as well as service provision and delivery procedures to highly diverse environments. Adaptability, an inherently challenging task, is further complicated by the fact that adaptation mechanisms should be generic, portable and interoperable so that they are themselves able to be applied to a diversity of entities in different circumstances. In this paper, we introduce a generic adaptation mechanism, which has been designed to address the aforementioned requirements, and we present its successful application in the context of a $3 \mathrm{G}$ service provision platform.

The rest of this document is organized as follows: At first, we present the environment where the proposed adaptation mechanism was integrated and applied, namely a software

\footnotetext{
${ }^{1}$ Part of the work presented in this paper has been performed in the frame of project IST-10206-MOBIVAS (http://mobivas.cnl.di.uoa.gr). The content of this paper expresses solely the opinions of the authors.
}

platform for provision of services over $3 \mathrm{G}$ mobile networks. We then discuss generic adaptation (with a particular focus on mobile service provision) and identify the fundamental requirements of a flexible adaptation mechanism as well as the critical choices involved in its design. Subsequently, we elaborate on the architecture, design and implementation of the proposed mechanism, perform an evaluation of its most significant features and illustrate its operation in the context of the $3 \mathrm{G}$ service provision platform. The last sections of this paper are dedicated to summary, conclusions and acknowledgements.

\section{A Framework for Value-Added Service Provision in 3G NETWORKS}

The proposed generic adaptation mechanism has been applied in the context of a distributed software platform for the provision and management of value-added services over mobile networks in $3 \mathrm{G}$ and beyond [3] [4]. The platform aims to address major issues regarding the deployment and management of services offered to users of next generation mobile networks. These applications are typically provided by third-party software vendors, commonly termed Value-Added Service Providers. The platform's functionality comprises automated procedures for service deployment that include appropriate reconfiguration of the underlying network for optimal service delivery. In addition to that, an intelligent context-aware mobile portal is offered to the end-user, where procedures like service discovery, downloading and adaptation are fully tailored to terminal capabilities, user preferences and network characteristics. The single, generic adaptation mechanism presented in this paper is re-used for all the above-mentioned customization actions.

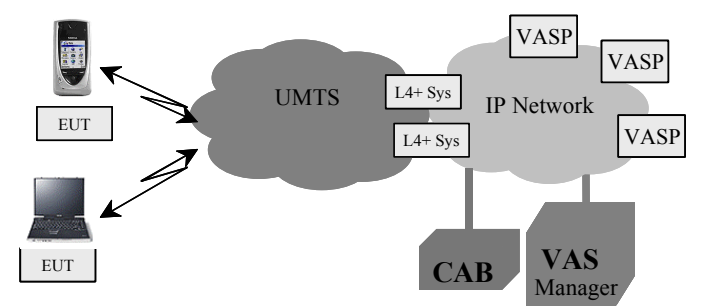

Figure 1. Architecture for flexible service provision in 3G networks. The architecture of the platform is depicted in Figure 1. The main components of this architecture are the following:

The Value-Added Service Manager (VASM) is the central platform component in that it co-ordinates the entire service provision and management process. It includes modules that undertake on-line service deployment by VASPs, network reconfiguration, maintenance of service- and user-related data in suitable databases and repositories, as well as customized service discovery, downloading and adaptation. The latter functions and in general all interactions with the user 
including the portal functions and the control of the adaptation procedures (e.g., issuing requests to the adaptation module) are handled by an internal module of the VASM, called User Interaction Management Module (UIMM). The adaptation module that implements the proposed adaptation mechanism is an autonomous part of the VASM. The UIMM acts as a client of this module during the adaptation procedure.

The Metering Devices (MDs) [5] are enhanced IP routers that are capable of providing QoS (e.g., via DiffServ) and monitor specific traffic flows. The MDs generate metering records that are necessary for billing.

The Charging, Accounting and Billing (CAB) [6] system is responsible for producing a single user bill for service access and apportioning the resulting revenue between the involved business players. This is accomplished by collecting metering data from standard 3G core network elements as well as MDs. The Mobile Terminal Software (MTS) includes functionality such as service downloading management, GUI clients for service discovery and selection, capturing of event notifications as well as service execution management. The MTS is able to identify and communicate to the VASM information useful to the adaptation mechanism, like terminal capabilities and user location.

3. Generic adaptation in Mobile Service Provision Adaptability in mobile computing is usually referred to as the ability of an entity (typically an application or a function) to dynamically modify its state and behavior according to its context. The context may include user preferences, terminal capabilities, network characteristics, user location and status (e.g., activity, mood) and can be expressed in parameters that vary significantly over time and space.

Adaptation to context, usually involves the following steps:

1. Context identification, which corresponds essentially to collecting the appropriate information from the current environment.

2. Decision on the most suitable (given the current context) transition to another state/behavior of the entity.

3. Application/activation of the selected transition.

Steps 1 and 3 above are inherently dependent on the particular entity instance and thus a mechanism covering all cases for these tasks cannot be entirely entity-independent. On the contrary, task 2 may be essentially reduced to generic, entityagnostic matching procedures. This paper introduces a generic mechanism for identifying the optimal choice for the state/behavior of an adaptable entity (e.g., version of application that should be downloaded to the terminal, components that should be included in the application package).

It is worth noting that an important aspect that has not been attracted the appropriate attention in the design of current adaptation systems is that the different adaptation phases should be, to the extent possible, decoupled from each other. This way, the functionality developed for each phase can be re-used for adapting various types of entities in diverse environments (e.g., ranging from web page content customization to dynamic composition of Java applications).

\subsection{Adaptation Requirements}

Developing a generic adaptation mechanism, suitable for highly dynamic environments, like mobile networks and services in $3 \mathrm{G}$ and beyond, is an excessively demanding task. The fundamental requirements for such a mechanism are identified below.

- Support for arbitrary complexity. The adaptation mechanism should support elements, like profiles and algorithms, of arbitrary complexity.

- Independence from particular types of profiles and algorithms. The algorithms that are used for profile matching should be loaded dynamically at run-time and thus should not be a static part of the adaptation mechanism. That is, any logic pertaining to specific types of profiles and algorithms should not be hard-coded in the adaptation system. Violating this would hamper scalability, since the support of a variety of adaptation data and processing logic would be achievable only by increasing the size and complexity of the system.

- Interoperability and portability. Ideally, the adaptation system should not only handle disparate data and algorithms, but also enable its seamless integration in a variety of environments. System modularity and decoupling from context are vital to the accomplishment of this goal. Another important parameter is compliance with international standards that are widely supported by industry players.

- Efficiency. The introduction of adaptation functionality should not compromise the performance of existing systems and services. Issues that require particular care are resource consumption in limited capabilities mobile terminal devices as well as congestion avoidance in lowbandwidth wireless network links.

\subsection{Design issues in dynamic feature negotiation and adaptation}

The present section provides a short discussion of certain general issues critical to dynamic feature negotiation and adaptation, pertaining to any context. However, we also focus on the case of $3 \mathrm{G}$ services and the solutions we propose for it.

\subsubsection{Data exchange mechanism}

During the adaptation procedures, profile data needs to be exchanged between various entities. Profile information exchange is particularly interesting, when it takes place across administrative boundaries. In this case, open, standardized technologies should be used so that interoperability is achieved. In the service adaptation approach presented herein, we use the CC/PP Exchange Protocol [8], a mechanism based on the HTTP/1.1 extension framework, for transporting terminal capability data from the terminal to server-side components, which is also support by the 3GPP MExE 
specification [9]. User and service profiles are retrieved ondemand from the corresponding databases maintained by the service provision platform operator. Network capabilities, on the other hand, should be obtained from the underlying network infrastructure. This task can be accomplished by the use of existing open APIs (e.g., OSA, JAIN) and may require their extension or even the definition of new ones.

\subsubsection{Adaptation mechanism}

The mechanism used for adaptation can follow several different models, namely selection, transformation and hybrid adaptation.

- Selection [10] is the process of choosing between a number of distinct, predefined alternatives. It requires that all the possible outputs of the adaptation function can be exactly predicted and statically defined a priori.

- Transformation [10] is used when the possible output of the adaptation function cannot be predicted with a degree of accuracy that enables its classification in distinct categories. Thus, when this approach is adopted, the adaptation function forms its output by dynamically combining appropriate entities on the fly.

- Hybrid adaptation is a combination of the two paradigms described above. As in selection, there is a pre-existing number of alternatives. These alternatives are, however, highly customizable, so that the final output of the adaptation function is a result of transforming and combining them with suitable entities.

In the special case of $3 \mathrm{G}$ service provision, adaptability could be applied to procedures like service discovery, selection of application variants or dynamic synthesis of a service from different components. All three of the aforementioned approaches are supported by the proposed mechanism, however, we believe that selection and hybrid adaptation are more appropriate, since the existence of predefined (and customizable, when using hybrid adaptation) alternatives simplifies user interactions and avoids overwhelming the enduser with an extensive number of possible choices.

\subsubsection{Location of adaptation function}

A crucial design decision regarding an adaptation system concerns the placement of the adaptation function, especially in environments following the dominant client/server paradigm. Two apparent alternatives are placing this function on the server or the client, respectively. An approach similar to the transparent negotiation [11], proposed for dynamic adaptation of web content, could be considered, where delegating part of the negotiation logic to client avoids the transport overhead of including client capabilities with every request.

As far as service provision adaptation in mobile networks is concerned, however, we have adopted a scheme, which is server-based. The client should be able to communicate to the server its capabilities and probably also certain characteristics of the underlying network, but the actual task of adaptation is performed at the server-side. This choice was made since the information required for this task can be more efficiently collected and retrieved by a fixed network server and namely the service provision platform. It would be highly costly in terms of network traffic to transfer all the necessary data (e.g., application, user, network profiles) to the terminal over the wireless link. Moreover, adaptation processing requires resources that are usually not available in limited capabilities terminals. The proposed, server-based approach has the overhead of having the terminal capability information conveyed to the server over the air, which is nevertheless not very high if the part of the terminal profile that is not subject to change over time (e.g., hardware characteristics of a specific terminal device) is replaced by a fixed network address (e.g., URL) from where the corresponding information can be retrieved.

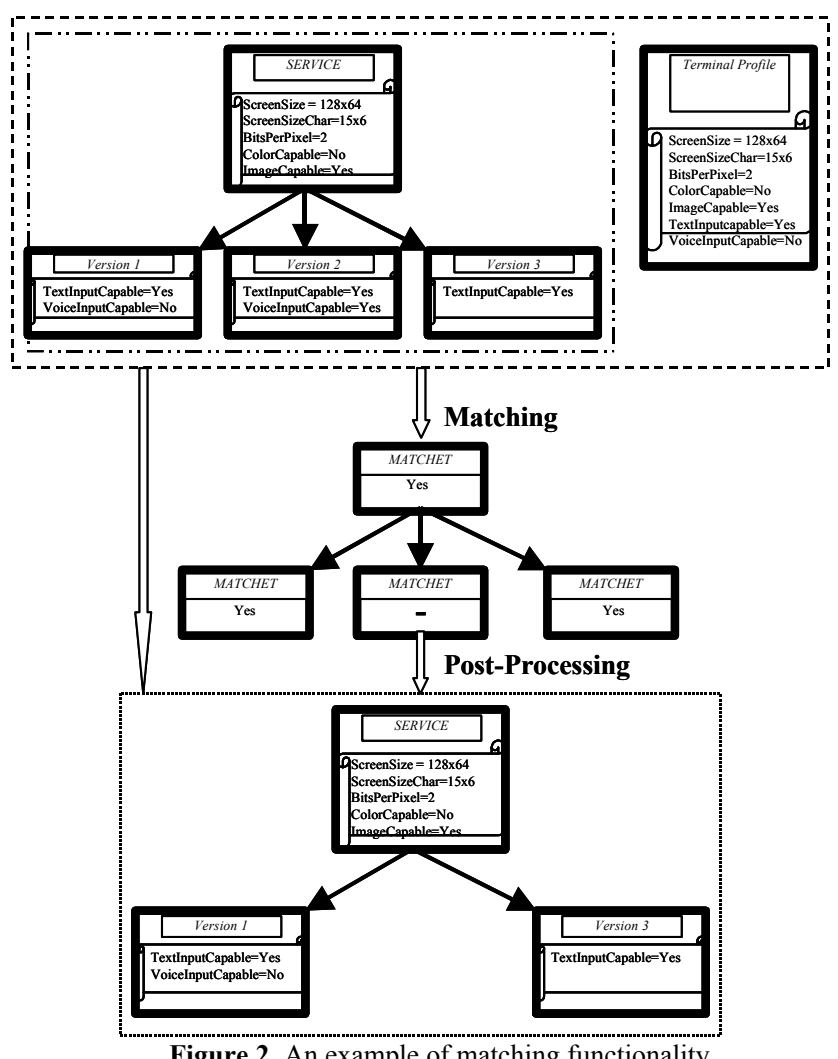

\subsection{Generic adaptation mechanism}

\subsubsection{Overview}

The adaptation system takes as input two profiles, called the adaptor and the adaptee. The adaptor is a profile encapsulating context information that is used for adaptation (e.g., for $3 \mathrm{G}$ service provision the adaptor could represent the capabilities of a terminal, the preferences of a user or the characteristics of a network). The adaptee denotes the entity that needs to be customized according to the adaptor data. A single adaptee may also describe entity collections and/or hierarchies with arbitrary size, structure and semantics. 
Essentially, the adaptee profile has the form of a tree. Each node in the tree represents a customizable unit that is identified by name and type and could be part of a larger structure. Moreover, every node contains a profile of adaptor type, that is a profile that indicates the capabilities/preferences/requirements of the corresponding unit pertaining to the specific adaptation criteria expressed by an adaptor. A special degenerated case would be the adaptee representing an atomic, non-decomposable entity and thus takes the form of a tree with a single node. Each adaptee profile instance aggregates an object that encapsulates the matching algorithm (we do not use a declarative way to represent matching criteria, since we believe that directly developing the corresponding code can be a more straightforward and easy solution in the majority of cases). This is due to the fact that the matching algorithm may vary not among various types of attributes, (e.g., String, ScreenSize) but also between different instances of the same attribute type.

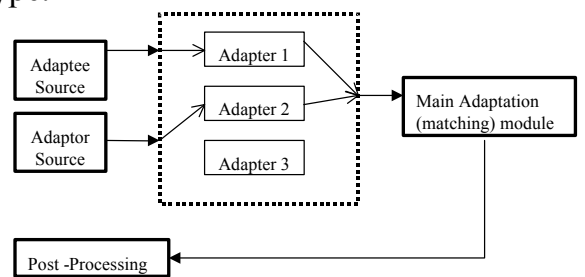

Figure 3. Architecture of the proposed adaptation system.

For instance, in a situation where a $3 \mathrm{G}$ mobile user wants to view which services are accessible from his/her current terminal, the service provision system should retrieve the list of available services (from an application database/repository) and filter it according to the capabilities of the user's terminal. We assume that each service may come in multiple versions and that service- and version-specific terminal requirements (together with information on where the corresponding matching algorithms may be retrieved from) are obtainable from the service database. In this case, the adaptor would be the terminal profile, while the adaptee would comprise a list of services, with each service typically consisting of a list of versions. This information would be arranged in a tree (Figure 2), with every node containing identification information for the service/version it represents, as well as the corresponding terminal requirements.

The output of the system is a customized version of the adaptee. When selection is applied, the only information returned is the list of the alternative representations/versions of the customizable entity that match the particular environment. In case of hybrid adaptation, every item in the list is annotated with a declarative description of the additional customizations that are appropriate. Thus, the adaptation result has the form of a tree. In the context of the proposed system, this tree is called matchet. An example matchet for the example described above is depicted in

\subsubsection{Architecture}

The architecture of the proposed generic adaptation system is depicted in Figure 3.

The overall adaptation procedure is divided into three distinct phases: Pre-processing, main adaptation (or matching) and post-processing. The latter phase, however, is not strictly part of the overall generic adaptation mechanism, and would typically reside in an environment-specific client, as explained later in this paragraph.

The pre-processor is fed with the adaptee and adaptor profiles and undertakes to transform them to a format suitable for processing by the main adaptation function. The latter accepts as inputs hierarchical profiles in the form of Java objects. The class diagram of Figure 4 depicts the representation of profiles as Java classes in our implementation. Notably, the adaptee and adaptor profiles are instances of the same class (Profile). Profiles are instances of profile elements and consist of single profile attributes and sub-profiles (this is an application of the Composite design pattern [12]). The interface of each profile element consists of the match method, which encapsulates the algorithm for determining if a profile element object matches another profile element object of the same type.

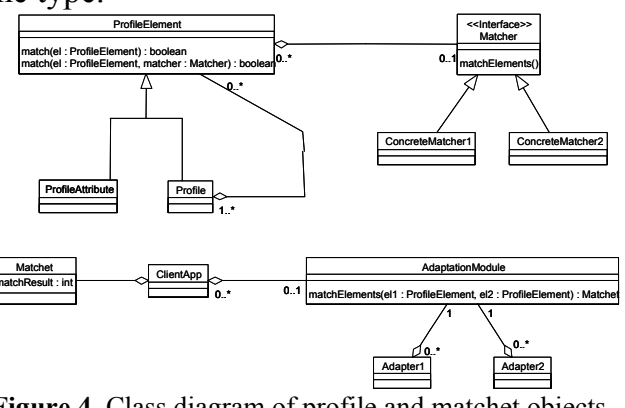

Alternatively, the adaptation algorithm may be encapsulated in a Matcher object that is aggregated in a ProfileElement instance and dynamically loaded. This way, new matching algorithms for specific attributes can be introduced without the need to modify (or recompile) the code of the ProfileElement classes that represent those attributes (this is an application of the Strategy design pattern [12]).

The pre-processor identifies the current format of the profiles and either forwards them directly to the matching function (if they are in the right format), or dispatches them to the appropriate module for the required transformation (the design of the pre-processor makes use of the Facade and Adapter patterns [12]). In our current implementation we support RDF/XML as encoding formats. Naturally, adapters for other formats can be seamlessly plugged in the system, enhancing its functionality without affecting the rest of the components.

The main adaptation (matching) module receives the adaptee and adaptor profile trees in the form of Java objects, performs the appropriate matching and returns the corresponding matchet. The matching is carried out by traversing the adaptee hierarchy and by comparing the adaptor profile with the 
requirements/preferences profile in each node of the adaptee tree. The latter comparison is performed by recursive calls to the match method of each profile element or by using the matchElements method of the appropriate Matcher. Thus, the matching module is not required to be aware of the profile element internals (content, matching algorithm). Finally, the post-processor produces the final adapted entity.

It is worth noting that, while the pre-processing and matching modules are agnostic of the content and semantics of the adaptor and adaptee profiles, the post-processing function is dependent on the above parameters. Thus, when concerning the physical placement of these modules in the context of a system using the proposed adaptation mechanism, the preprocessing and main adaptation modules would reside in an autonomous, generic adaptation server, while the postprocessing module would be plugged in the adaptation client. The latter would typically be the entity that requires the customization of the adaptee profile. For instance, in the 3Gservice provision platform case, the post-processing module resides in the VASM and in particular in the UIMM, which is also the source of the adaptee profile.

\subsubsection{Evaluation of the proposed solution}

The proposed mechanism has a number of important features:

- It is able to handle arbitrarily complex hierarchical profile data structures, as well as any matching algorithm.

- The adaptation system code is independent of particular profiles, since the latter are encapsulated in ProfileElement (i.e., Profile and ProfileAttribute) objects.

- Adaptation algorithms are dynamically loaded at runtime. Thus, algorithms unknown or not implemented at the time of the development of the adaptation system can be seamlessly used.

- The system is compatible with the standard mechanisms of content representation and negotiation (CC/PP, $\mathrm{RDF} / \mathrm{XML}$ ) defined by the $\mathrm{W} 3 \mathrm{C}$ and adopted by the 3GPP.

- The adaptation function is placed on the server side and thus in principle does not incur excessive overhead in system resources like processing power and memory.

\subsubsection{Application of the proposed system for $3 \mathrm{G}$ service provision}

In the service provision platform described in Section 2, the proposed adaptation mechanism is applied for procedures like customization of listings of available services (in this case selection is used) as well as for determining per request the components of an application that should be packaged by the VASM and downloaded by the user terminal (hybrid adaptation).

Both procedures require matching of application profiles with terminal, user and network profiles. Thus, service profiles are the adaptees, while terminal, user and network profiles undertake the adaptor role. Adaptation includes three cascading matchings, one for each of the three adaptors. The UIMM is the adaptee and the adaptor source for all three cases. However, different types of profiles come from different origins: Service and user profiles are obtained by querying the corresponding databases, maintained by the platform operator, terminal capabilities are sent to the VASM by the mobile terminal, while network characteristics can be retrieved from the underlying infrastructure through appropriate open APIs (further elaboration on these APIs and their exact definition is beyond the scope of the present contribution). The pre-processing and matching functions are handled by a separate, autonomous module of the VASM, the adaptation module, while the context-dependent postprocessing function is located in the UIMM.

\section{CONCLUSIONS}

Emerging mobile communication systems from $3 \mathrm{G}$ and beyond are expected to pave the path towards the vision of an abundance of functionality-rich, highly customizable service offerings that end-users will be able to access from any terminal and over any network. The realization of this ambitious goal requires generic adaptation mechanisms that are independent of the data and algorithms used for the adaptation function to cater for the largely diverse wireless communications environment. In the present paper we introduced a generic adaptation mechanism that aims to address the above requirements. The proof of concept and feasibility of this mechanism has been demonstrated by its implementation in Java and its successful integration into a software platform for the provision and management of services over $3 \mathrm{G}$ networks.

\section{REFERENCES}

[1] UMTS Forum Reports No. 9, 10. 11, available from http://www.umtsforum.org/.

[2] N. Houssos, M. Koutsopoulou, S. Schaller, "A VHE architecture for advanced value-added service provision in 3rd generation mobile communication networks", Proceedings of the Globecom 2000 Workshop on Service Portability and Customer Services Environments, San Francisco, USA, November 2000, pp.69-79.

[3] N. Houssos, et al., "Value-Added Service Management in 3G networks", IEEE/IFIP Networks Operations and Management Symposium (NOMS 2002), 15-19 April 2002, Florence, Italy, pp. 529545.

[4] S. Panagiotakis, N. Houssos, A. Alonistioti, "Integrated Generic Architecture for Flexible Service Provision to Mobile Users", PIMRC 2001, San Diego, California, USA, 30/9-3/10 2001.

[5] T. Harbaum, et al., "Layer 4+ switching with QoS support for RTP and HTTP", pro ceedings of the Globecom 1999, Rio de Janeiro, Brazil, December 1999.

[6] M.Koutsopoulou, C. Farmakis, E. Gazis, "Subscription Management and Charging for Value Added Services in UMTS Networks", IEEE Semiannual Vehicular Technology Conference VTC2001, May 2001, Rhodes, Greece.

[7] RDF: Resource Description Framework home page, http://www.w3.org/RDF/.

[8] CC/PP working group home page, http://www.w3.org/ Mobile/CCPP/.

[9] 3G TS 23.057: "Mobile Station Application Execution Environment (MExE)".

[10] Kynn Bartlett, CC/PP, http://www.ccpp.org.

[11] K. Hoeltman, A. Mutz, "Transparent Content Negotiation in HTTP", IETF RFC 2295.

[12] Erich Gamma et al., "Design Patterns: Elements of Reusable Object Oriented Software", Addison Wesley Longman, Inc. 